\title{
The SPES project of INFN: Facility and detectors
}

\author{
G. DE Angelis ${ }^{1}$ for the SPES Collaboration \\ G. Prete ${ }^{1}$, A. Andrighetto ${ }^{1}$, M. Manzolaro ${ }^{1}$, S. Corradetti $^{1}$, \\ D. Scarpa ${ }^{1}$, M. Rossignoli ${ }^{1}$, A. Monettit ${ }^{1}$, M. Lollo $^{1}$, \\ M. Calderolla ${ }^{1}$, J. Vasquez ${ }^{1}$, D. Zafiropoulos ${ }^{1}$, L. Larchiapone $^{1}$, \\ D. Benini ${ }^{1}$, P. Favaron ${ }^{1}$, M. Rigato ${ }^{1}$, R. Pegoraro ${ }^{1}$, D. Maniero ${ }^{1}$, \\ L. Calabretta ${ }^{2}$, M. Comunian ${ }^{1}$, M. Maggiore ${ }^{1}$, A. Lombardi $^{1}$, \\ L. Piazza ${ }^{1}$, A.M. Porcellato ${ }^{1}$, C. Roncolato ${ }^{1}$, G. Bisoffi ${ }^{1}$, \\ A. Pisent ${ }^{1}$, A. Galatà ${ }^{1}$, M. Giacchini $^{1}$, G. Bassato ${ }^{1}$, S. Canella $^{1}$, \\ F. Gramegna ${ }^{1}$, J. Valiente ${ }^{1}$, J. Bermudez ${ }^{1}$, P.F. Mastind ${ }^{1}$,

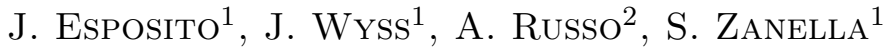

${ }^{1}$ INFN-LNL, Laboratori Nazionali di Legnaro, I-35141, Legnaro (PD), Italy ${ }^{2}$ INFN-LNS, Laboratori Nazionali del Sud, I-95123, Catania, Italy

\begin{abstract}
The SPES Radioactive Ion Beam facility at INFN-LNL is presently in the construction phase. The facility is based on the Isol (Isotope separation on-line) method with an UCx Direct Target able to sustain a power of $10 \mathrm{~kW}$. The primary proton beam is provided by a high current Cyclotron accelerator with energy of 35-70 MeV and a beam current of $0.2-0.5 \mathrm{~mA}$. Neutron-rich radioactive ions are produced by proton induced Uranium fission at an expected fission rate of the order of $10^{13}$ fissions per second. After ionization and selection the exotic isotopes are re-accelerated by the ALPI superconducting Linac at energies of $10 \mathrm{~A} \mathrm{MeV}$ for masses in the region $A=130 \mathrm{amu}$. The expected secondary beam rates are of the order of $10^{7}-10^{9}$ pps. Aim of the SPES project is to provide a facility for high intensity radioactive ion beams for nuclear physics research as well as to develop an interdisciplinary research center based on the cyclotron proton beam.
\end{abstract}

This is an Open Access article distributed under the terms of the Creative Commons Attribution License 4.0, which permits unrestricted use, distribution, and reproduction in any medium, provided the original work is properly cited 


\section{Introduction}

The aim of the SPES project is to provide high intensity and high-quality beams of neutron-rich nuclei to perform forefront research in nuclear structure, reaction dynamics and interdisciplinary fields like medical, biological and material sciences. SPES is a second generation Isol radioactive ion beam facility. It represents an intermediate step toward the future generation European Isol facility EURISOL [1]. The SPES project is part of the INFN Road Map for the Nuclear Physics; it is supported by the Italian national laboratories LNL (Legnaro) and LNS (Catania). It is based on the ISOL method with an UCx Direct Target able to sustain a power of $10 \mathrm{~kW}$. The primary proton beam is delivered by a Cyclotron accelerator with an energy of more then $40 \mathrm{MeV}$ and a beam current of $200 \mu \mathrm{A}$. Neutron-rich radioactive ions will be produced by Uranium fission at an expected fission rate in the target of the order of $10^{13}$ fissions per second. The exotic isotopes will be re-accelerated by the ALPI superconducting Linac at energies of $10 \mathrm{~A}$ $\mathrm{MeV}$ and higher, for masses in the region of $A=130 \mathrm{amu}$, with an expected rate on the secondary target of $10^{7}-10^{9}$ pps.

\section{Challenges of the SPES project}

Our knowledge about the structure of nuclei is mostly limited to nuclei close to the valley of stability or nuclei with a deficiency of neutrons. Only recently the availability of beams of unstable ions has given access to unexplored regions of the nuclear chart, especially on the neutron-rich side. Starting from a nucleus on the stability line and adding successively neutrons, one observes that the binding energy of the last neutron decreases steadily until it vanishes and the nucleus decays by neutron emission. The position in the nuclear chart where this happens defines the neutron drip line. It lies much farther away from the valley of stability than the corresponding drip line associated with protons, owing the absence of electrical repulsion between neutrons. The location of the neutron drip line is largely unknown as experimental data are available only for nuclei with mass up to around 30. The interest in the study of nuclei with large neutron excess is not only focused on the location of the drip line but also on the investigation of the density dependence of the effective interaction between the nucleons for exotic $\mathrm{N} / \mathrm{Z}$ ratios. In fact, changes of the nuclear density and size in nuclei with increasing $\mathrm{N} / \mathrm{Z}$ ratios are expected to lead to different nuclear symmetries and new excitation modes. While in the case of some 
very light nuclei a halo structure has been identified, for heavier nuclei the formation of a neutron skin has been predicted. The evolution of nuclear properties towards the neutron drip line depends on how the shell structure changes as a function of neutron excess. This evolution has consequences on the ground state properties of the nuclei and on the single-particle and collective excitations. In particular, studies of neutron-rich nuclei beyond the doubly magic ${ }^{132} \mathrm{Sn}$ are of key importance to investigate the single-particle structure above the $\mathrm{N}=82$ shell closure and find out how the effective interaction between valence nucleons behaves far from stability. New modes of collective motion are also expected in connection with the formation of a neutron skin, namely oscillation of the skin against the core, similar to the soft dipole mode already identified in the case of very light halo nuclei. Presently, neither the thickness nor the detailed properties of the neutron skin of exotic nuclei are known. This information is needed to enable a quantitative description of compact systems like neutron stars, where exotic nuclei forming a Coulomb lattice are immersed in a sea of free neutrons, a system which is expected to display the properties of both finite and infinite (nuclear matter) objects. At the energy of SPES, it will be possible to address important questions related to the study of neutron-rich matter such as nuclear forces, level density, viscosity, barrier, neutron pairing and collective modes. With the high intensity beams delivered by SPES, a challenging and broader range of studies in nuclear spectroscopy and reaction mechanism will be performed. Interesting areas where new data will be collected are those in the very neutron rich regions, where shell evolution is an issue. Effects of how the pairing interaction is modified in the nuclear medium will receive significant inputs by measurements of multi-nucleon transfer reactions to specific nuclear states. Effects of rotational damping in the decay of high energy levels, for instance the dynamical dipole emission, will be studied by changing the N/Z of projectile and target. Neutron rich nuclei will be used to investigate the tunneling process in sub-barrier fusion reactions in presence of very positive $\mathrm{Q}$-values, an issue interesting also for astrophysics.

\section{The SPES radioactive ion beam facility}

SPES is an INFN project involving the two national laboratories, LNL and LNS and other INFN sites in Italy, based on the Isol technique for radioactive beam production. A primary beam provided by a driver accelerator induces nuclear reactions inside a thick target. The reaction products are extracted 
from the target by thermal process due to the high temperature of the target-ion source system (exceeding $2000^{\circ} \mathrm{C}$ ). Once reached the $1^{+}$source, the products are ionized and extracted. After an isotopic selection and a further ionization to $\mathrm{n}^{+}$the exotic beam is injected into a re-accelerator. SPES choice will use a proton beam to induce fission on a UCx target (Direct Target). The driver is a $70 \mathrm{MeV}$ proton cyclotron with two exit ports for a total current of $750 \mu \mathrm{A}$. Two production Isol targets are planned to be installed and operated alternatively. As the cyclotron can supply two beams at the same time, a second independent facility can be operated in parallel. The high intensity proton beam will be used to produce radioisotopes for nuclear medicine as well as a neutron source. The expected neutron production at SPES exceeds $10^{14} \mathrm{n} / \mathrm{s}$ with energy up to $70 \mathrm{MeV}$ and a peak between 0.1 and $10 \mathrm{MeV}$ according to target and moderator. The proton beam of the SPES cyclotron is suited for the production of new radioisotopes for medical applications. Taking advantage of the high energy and current, it opens up the possibility to produce ${ }^{82} \mathrm{Sr},{ }^{67} \mathrm{Cu}$, and ${ }^{47} \mathrm{Sc}$ to be used in therapy and diagnostics. A pictorial sketch of the facility is shown in figure 1 .

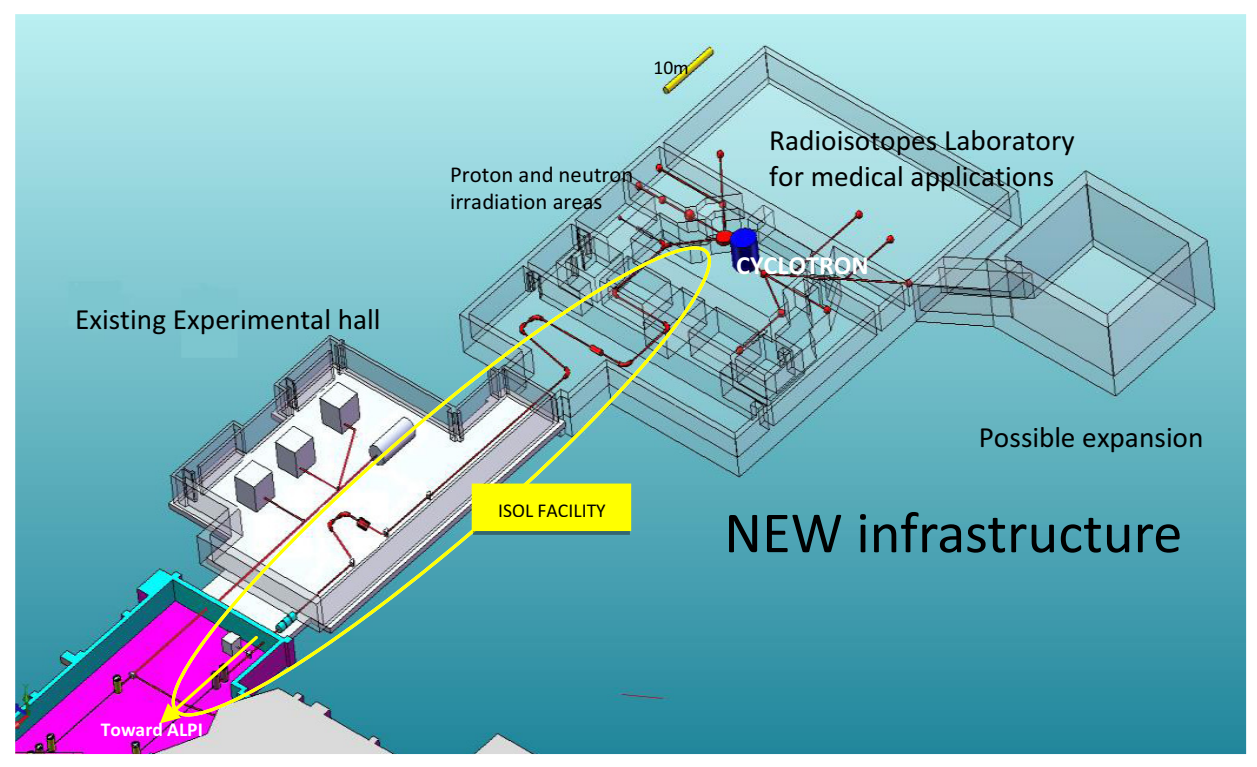

Figure 1: Pictorial sketch of the SPES facility. The ALPI superconductive linac (not shown) is on the left side. 


\subsection{The ISOL front-end}

The Isol front-end is the system which couples the proton beam with the UCx target, the ion source and the first part of the transport line of the exotic beam. The whole system was completed and is in operation at LNL for off-line tests. he Direct Target follows the basic design of the CERN ISOLDE one but represents an innovation in term of capability to sustain the primary beam power. The design is carefully oriented to optimize the radiative cooling, taking advantage of the target system's high operating temperature, which is of the order of $2000^{\circ} \mathrm{C}$. The SPES target is designed in order to maximize the release efficiency and the heat dissipation. It consists of multiple thin disks housed in a cylindrical graphite box. It is composed of $7 \mathrm{UCx}$ disks (diameter and thickness of 40 and $1.3 \mathrm{~mm}$, respectively), appropriately spaced in the axial direction in order to dissipate, by thermal radiation, the average power of $8 \mathrm{~kW}$ due to the energy release of the proton beam which, passing through them, induces nuclear reactions. The graphite box is housed inside a tubular hollow tungsten ohmic heater. This allows for a better thermal control of the target operation. An extensive simulation study of the target behaviour for thermal and release properties is at the basis of the target-ion-source design. Experimental work to benchmark the simulations was carried out in collaboration with HRIBF [2] , the Oak Ridge National Laboratory ISOL facility (USA). Partial results on the measured production rates (and on the expected rates of the reaccelerated SPES beam) are shown in figure 2 .

\subsection{The ion-source system}

The interaction of the proton beam with the UCx target will produce neutron-rich fission fragments subsequently extracted by thermal motion and ionized at $1^{+}$charge state by the Isol source directly connected with the production target. The hot-cavity ion source chosen for the SPES project was designed at CERN (ISOLDE) [3]. The source has the basic structure of the standard high temperature ion sources employed for on-line operation. The ionizer cavity is a thungsten tube (34 $\mathrm{mm}$ length, $3 \mathrm{~mm}$ inner diameter and $1 \mathrm{~mm}$ wall thickness) resistively heated to near $2000^{\circ} \mathrm{C}$. The isotopes produced in the target diffuse in the target material and then effuse through the transfer tube into the ionizer cavity where they undergo surface or laser ionization. The Surface ionization process can occur when an atom comes into contact with a hot metal surface. In the positive surface ionization, the transfer of a valence electron from the atom to the metal surface is ener- 


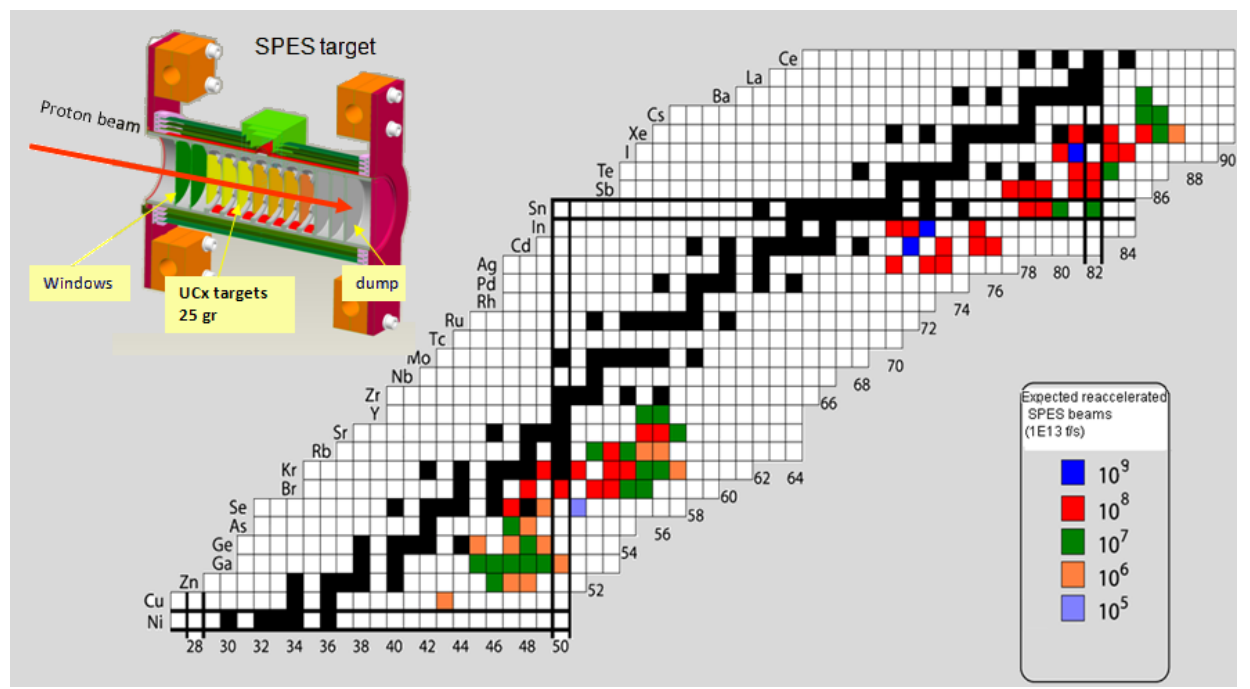

Figure 2: Expected beam on target intensities. Experimental data scaled to: 0.2 $\mathrm{mA}$ proton current, $2-5 \%$ transport efficiency. ISOL target operated at $2000^{+} \mathrm{C}$, ionization and extraction with $1^{+}$plasma source.

getically favorable for elements with an ionization potential lower than the work function of the metal. For alkalis and some rare earth elements, high ionization efficiencies can be achieved using the surface ionization technique. Such $1^{+}$source has good efficiency and selectivity for the elements as Rb, Cs, Ba. For most of the other elements, the laser resonant photo-ionization, using the same hot cavity cell, is a powerful method to achieve sufficiently selected ionization. This technique, now under development, has been implemented with the aim to produce beams as pure as possible (chemical selectivity) also for metal isotopes. To ionize elements with high ionization potential, as rare gasses, the plasma source is needed. This source ionizes all the elements without any selectivity. At SPES both surface and plasma sources have been developed and are in operation at the off line test stand. The laser source is presently under development.

\subsection{Beam selection and transport}

The first mass selection is performed by a Wien Filter with 1/100 mass resolution, installed just after the first electrostatic quadrupole triplet inside the production bunker with the aim to confine the larger part of radioactivity inside the high shielded area. The transfer line toward ALPI is equipped with several beam handling systems to purify the beam. A Beam Cooler 
and a High Resolution Mass Separator (HRMS) with 1/20000 mass resolution will be installed inside the new building. Special efforts have been dedicated to design a mass spectrometer with an effective mass resolution of at least 1/20000. Such design takes advantage of a Beam Cooler, to improve the beam emittance, and of a $260 \mathrm{keV} \mathrm{HV}$ platform on which the mass separator is mounted. The mass separator is a scaled-up version of the separator designed for the CARIBU facility [4] in ANL (USA). In the SPES configuration the calculated resolution is of the order of $1 / 20000$ constrained by an emittance of $3 \mathrm{~mm}$ mrad and an energy spread of $1.3 \mathrm{eV}$. Such high selectivity results in an advantage also for the safety issue, reducing the contaminations along the beam transport area, the re-accelerator and the final target location. Before the injection in the ALPI superconducting Linac it is necessary an increase of the charge state from $1^{+}$to $\mathrm{n}^{+}$. This is performed by means of a Charge Breeder. The SPES Charge Breeder is based on ECR method [5] and aims to produce ions with A/q less than 7 at $A \approx 130$. The design and construction of the Charge-Breeder will be performed in the framework of the SPES-SPIRAL2 collaboration at LPCS (Grenoble, France) following an up-graded version of the Phoenix booster in operation at LPCS. After the Charge Breeder a second mass separator with 1/1000 mass resolution, will be installed to clean the beam from the contaminants introduced by the Charge Breeder itself. The same basic configuration used for the HRMS is adopted. Finally an RFQ pre-accelerator will increase the beam energy to match the ALPI acceptance. The layout of the radioactive beam line is showed in figure 3 .

\subsection{Exotic beam reacceleration}

The secondary beams are injected into the ALPI superconducting Linac by a new RFQ that will operate in a CW mode ( $100 \%$ duty factor) at a resonant frequency of $80 \mathrm{MHz}$. This frequency is the same as that of the superconducting structures of the lowest energy section of ALPI. The injection energy of the ions is set to $5.7 \mathrm{keV} / \mathrm{u}$. This choice is a compromise between the desire to reduce the ion energy to simplify the low energy injection and the RFQ bunching section design and the need to increase the beam rigidity in the $1^{+}$transport line to reduce space charge effects. The extraction energy of the RFQ is set to $727 \mathrm{keV} / \mathrm{u}$ to optimize the beam dynamics of the superconducting linac ALPI. ALPI is the superconducting heavy ion Linac in operation at LNL since the early nineties. It is composed by three sections of quarter wave resonators (QWR) for a total of 80 cavities installed and operates routinely at an equivalent acceleration voltage of $50 \mathrm{MV}$. The Linac 


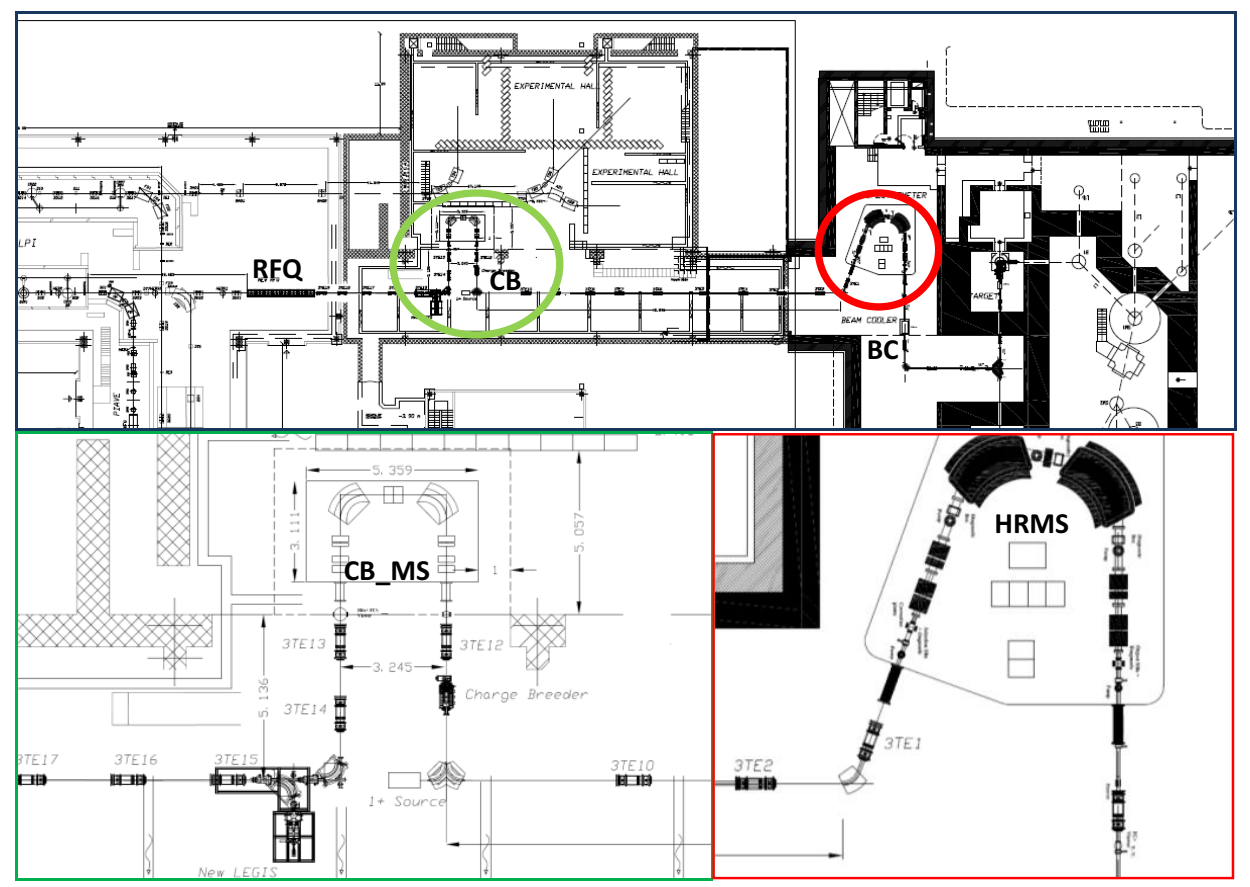

Figure 3: Layout of radioactive beam handling and transfer line. High resolution mass separator and the Charge Breeder mass separator are shown in the lower panels.

is constructed in a bended configuration: it is composed by two branches connected by an achromatic and isochronous U-bend. It uses three different kinds of cavities: Low Beta, Medium Beta and High Beta cavities, according to the different velocity along the acceleration path. The ALPI linac has gone through a continuous upgrade in the number and performance of its accelerating cavities, and consequently in the maximum achievable beam energy. In the last years the cavities of the medium energy QWR section have been upgraded using a new $\mathrm{Nb}$ sputtered coating in substitution to the original $\mathrm{Pb}$ sputtered layer. A further upgrade program is on the way, to improve the accelerating fields of the present QWRs and adding more cavities in the Low Beta section. The final equivalent voltage will exceed 70 MV going well beyond the Coulomb barrier for nuclear reactions involving mid-A projectiles and heavy targets. In figure 4 the expected beam energies are reported. 


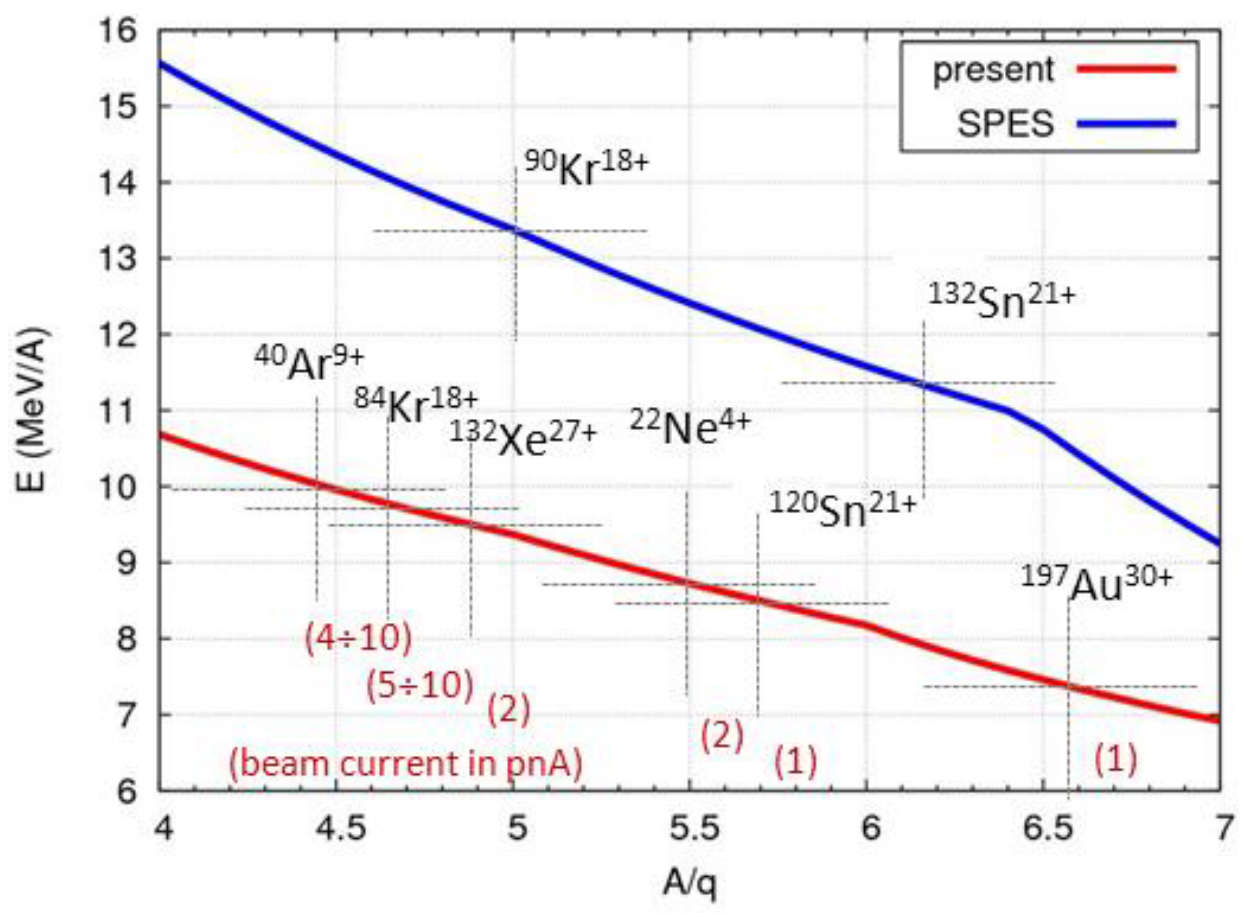

Figure 4: Beam energy performance of ALPI superconductive Linac.

\subsection{Status of the SPES project}

As already mentioned SPES is presently under construction at LNL. The building is at the excavation phase and will be completed at end of 2014. The cyclotron, provided by the BEST company in Canada, is under construction (the magnet is completed and the magnetic field was positively mapped). It is expected to be transferred to LNL, after the factory commissioning, in the second quarter of 2014. The authorizations to operate the cyclotron have been already obtained allowing the acceleration of the proton beam on non-fissile target at high power and testing the UCx target up to a current of $5 \mu \mathrm{A}$. A further authorization request to run the $\mathrm{UCx}$ target at design power will be presented to the Italian authorities at the end of this year. The physics design of the radioactive ion beam transfer line $\left(1^{+}\right.$and $\left.\mathrm{n}^{+}\right)$ is completed and the reshaping of the ALPI beam line in the experimental hall is initiated to allow the installation of the Charge Breeder and of the mass separator. The Isol front-end is completed and under operation in the off-line laboratory to test ion sources, assembly and controls. The first reaccelerated exotic beam is expected around 2018 [6]. 


\section{Detectors for the SPES RIB facility}

The scientific program of SPES has been developped through two dedicated workshops with the presentation of letters of intent from a large international scientific community and with plans to bring new instrumentation from all over the world. The scientific program for the low energy RIB (without reacceleration) has been focused on a beta decay station, a total absorption spectrometer and a detector for beta delayed neutrons. A time-of-flight multi-bunch spectrometer has been indicated for mass measurements. For the experimental campaign using reaccelerated RIBs several set-ups have been proposed. A new dedicated instrumentation (SPIDER) is under construction for Coulomb excitation measurements. For direct reaction measurements different approaches have been considered, based on Si multidetector arrays (TRACE-GASPARD) or on Active Targets (SPESACTAR) or using spectrometers (PRISMA or Helios like). Medium- and high-energy gamma ray studies have been proposed based on the novel high resolution scintillator array PARIS. A new neutron detector (NEDA) has been proposed for n-tagging and a novel detector for heavy ion identification (FAZIA) for the study of the equation of state for asymmetric nuclear matter. The AGATA and Galileo high resolution gamma ray spectrometers have been indicated for nuclear structure studies. All developments are well tailored to the program envisaged with the SPES beams and are coordinated within the international context by the EU SPIRAL2 PP program.

\section{References}

[1] www.eurisol.org

[2] D. Scarpa, L. Biasetto, S. Corradetti, M. Manzolaro, A. Andrighetto, S.Carturan, G. Prete, P. Zanonato and D.W. Stracener Eur. Phys. J. A (2011) 47

[3] M.Lindroos, CERN-AB-2004-086, (2004)

[4] Savard, G.; Pardo, R.; Baker, S.; Davids, C.; Levand, A.; Peterson, D.; Phillips, D.; Sun, T.; Vondrasek, R.; Zabransky, B.; Zinkann, G; Hyperfine Interactions, Vol. 199 Issue 1-3, (2011) 301

[5] T. Lamy, J.-L. Bouly, J.-C. Curdy, P. Sole, P. Sortais, T. Thuillier, J.-L. Vieux-Rochaz, and D. Voulot ; Rev. Sci. Instrum. 75, 1624 (2004)

[6] https://web.infn.it/spes/ 